\title{
Optimization Model of Load and Transportation for Prefabricated Construction Components

\author{
CHANG Chunguang $^{\mathrm{a}^{*}}$, WU Feifei ${ }^{\mathrm{b}}$, LIU Dan ${ }^{\mathrm{c}}$
}

School of Management, Shenyang Jianzhu University, Shenyang 110168, China

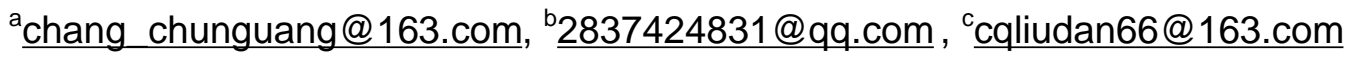

Keywords: Prefabricated construction components; transportation; cost; programming theory

\begin{abstract}
Low utilization rate of transport vehicle for prefabricated construction (PC) components is one important reason of the high transportation cost. On the basis of analyzing the geometrical characteristics of PC components and installation sequence, the vehicle load capability and principles are taken as constraint conditions, aiming to maximize load quantity, the optimization model of load and transportation for PC Components is established by programming theory. It can achieve reasonable division of bulk and organization of load transportation problem, control the load transportation cost. By comparative analysis in practice, it can be proved that the optimized load scheme is superior to the original one, it can reduce the number of transport vehicles used, and also significantly reduce the transportation cost. It will provide reference to the practice of transportation cost control of PC components.
\end{abstract}

\section{Introduction}

Prefabricated construction (PC) is different from cast-in-place construction. The cost of PC civil engineering cost includes component production costs, transportation costs, installation fees and so on [1]. The high transportation cost is one of the main reasons for the high cost of the prefabricated buildings. Due to the characteristics of the PC and the limitation of the engineering transportation, the loading rate and the space utilization rate of the vehicle are not reasonably utilized, which leads to the high transportation cost. In view of the above situation, how to use the vehicle load and space utilization rate of the transportation vehicle is a problem to be solved urgently.

From the perspective of loading and transportation, Liu Hengsong and Sun Yuanming take the Large-sized semicircular steel structures as example, introduce the preparation of transportation schemes and the actual operation [2]. From the perspective of transportation management, Wang Hongchun applies BIM technology to the construction industry's logistics management [3]. Evensen combines the traditional transport management with advanced C-ITS, providing a new ITS station [4]. Zhou Lixin applies the intelligent transportation technology to logistics management [5]. Fang Yuan and $\mathrm{Wu}$ Zhaotang study the composition of construction logistics costs [6].

Above research work provides the theoretical basis for the transportation cost control of the PC components. However, due to the particularity of the geometry of the assembly and particularity of the construction site environment, the traditional logistics transportation management theory should be further studied so as to be applied to the transportation of the PC components.

\section{Loading Scheme Optimization Model}

Variable Setting. Variables are set as follows.

$N_{A}, G_{A}, V_{A}$-number, total weight and volume of components to be installed in a certain period.

$x_{i j k}, g_{i j k}, L_{i j k}, W_{i j k}, H_{i j k}$ :-number, quality, length, width and thick of PC components on the $i$ th vehicle from the $j$ th type and by the $k$ th loading mode.

$L, W, H, G, V, h$-limited length, width, height, carrying capacity, volume, chassis height of the loaded vehicles.

$\lambda, \beta, \gamma^{\prime}$ - maximum length in each row by vertical and direct, flat and direct, flat and horizontal component loading mode.

$\beta^{\prime}, \gamma$ - maximum width in each row by flat and direct, flat and horizontal component loading mode. 
$a$ - spacing for flat or vertical position.

$c$ - distance from vehicle boundary to the component or safety distance between adjacent two rows. $S_{i k p}$ - number of rows or columns on the $i$ th vehicle by the $k$ th loading mode.

$m, n$ - required number of vehicles, number of PC component type.

$k$-loading mode, $k=1,2,3,4$,respectively, represent vertical and direct, vertical and horizontal , flat and direct,flat and horizontal;

$j^{*}$ - internal and external wall with doors or windows hole.

$z_{i}, Q_{j}$ - number of components on the $i$ th vehicle, the $j$ th kind components.

$Y$ - free space for all vehicles.

$s_{1}$ - number of components loaded per vehicle if components are calculated by maximum weight;

$s_{1}^{\prime}$ - number of columns loaded per vehicle if components are calculated by maximum volume.

$s_{1}$ - number of rows loaded per vehicle if components are calculated by maximum volume.

$s_{2}$ - number of components loaded per vehicle if components are calculated by maximum weight.

$H^{*}, L^{*}$ - thickness, length of component with largest volume.

$g^{*}$-weight of the component with largest quality.

Modeling Establishing. The transport vehicle loading optimization model is established as follows:

$$
\begin{aligned}
& \text { Min } Y= \sum_{i=1}^{m} \mathrm{f}\left(z_{i}\right) V-\sum_{i=1}^{m} \sum_{j=1}^{n} \sum_{k=1}^{4} x_{i j k} H_{i j k} W_{i j k} L_{i j k} . \\
& \text { s.t. } \quad S_{1}^{\prime}=(L-c) /\left(L^{*}+c\right) . \\
& S_{1}^{\prime \prime}=(W-2 c+a) /\left(H^{*}+a\right) . \\
& s_{1}=s_{1}^{\prime \prime} s_{1}^{\prime \prime} . \\
& s_{2}=\mathrm{INT}\left(G / g^{*}-0.5\right) . \\
& m=\mathrm{INT}\left[N_{A} / \min \left(s_{1}, s_{2}\right)+0.5\right] . \\
& 0 \leq \sum_{j=1}^{n} \sum_{k=1}^{4} x_{i j k} g_{i j k} \leq G,=1,2 \ldots ; i=1,2 \ldots, m . \\
& 0 \leq \sum_{j=1}^{n} x_{i j 1}\left(H_{i j 1}+a\right) \leq(W-2 c+a) S_{i 11},=1,2 \ldots ; i=1,2 \ldots, m . \\
& 0 \leq S_{i 11}(\lambda+c) \leq L+c, i=1,2 \ldots, m . \\
& 0 \leq \sum_{j=1}^{n} x_{i j 2}\left(H_{i j 2}+a\right) \leq L-2 c+a, i=1,2 \ldots, m . \\
& 0 \leq S_{i 31}(\beta+c) \leq L+c, i=1,2 \ldots, m . \\
& 0 \leq S_{i 32}\left(\beta^{\prime}+c\right) \leq W+c, \mathrm{i}=1,2 \ldots, m . \\
& 0 \leq S_{i 41}(\gamma+c) \leq L+c, i=1,2 \ldots, m . \\
& 0 \leq S_{i 42}\left(\gamma^{\prime}+c\right) \leq W+c, i=1,2 \ldots, m . \\
& S_{i 11}(\lambda+c)+S_{i 31}(\beta+c)+S_{i 41}(\gamma+c)+\sum_{j=1}^{n} x_{i j 2}\left(H_{i j 2}+a\right) \leq L, i=1,2 \ldots, m . \\
& 0 \leq \sum_{j=1}^{n} x_{i j 3}\left(H_{i j 3}+a\right) \leq(H-h+a) S_{i 31} S_{i 32}, i=1,2 \ldots, m . \\
& 0 \leq \sum_{j=1}^{n} x_{i j 4}\left(H_{i j 4}+a\right) \leq(H-h+a)^{*} S_{i 41} * S_{i 42}, i=1,2 \ldots, m . \\
& \sum_{i=1}^{m} \sum_{k=1}^{4} x_{i j k}=Q_{j}, j=1,2, \ldots, n . \\
& \sum_{i=1}^{m} \sum_{j=1}^{n} \sum_{k=1}^{4} x_{i j k}=N_{A} . \\
& z_{i}=\sum_{j=0}^{n} \sum_{k=1}^{4} x_{i j k}, i=1,2 \ldots, m . \\
& \mathrm{f}\left(z_{i}\right)=\left\{\begin{array}{l}
1, z_{i}>0 \\
0, z_{i}=0
\end{array}, i=1,2, \ldots, m .\right. \\
&
\end{aligned}
$$

In above model, formula (1) represents minimum rest space for all load vehicles; formula (2) to formula (6) are used to limit the maximum number of transport vehicles; formula (7) represents that the weight of loading components on the $i$ th vehicle is less than carrying capacity; formula (8) to 
formula (14) respectively represents width and length limit by four loading modes including load mode of vertical and direct, vertical and horizontal, flat and direct, flat and horizontal; formula (15) represents length limit of mixed loading; formula (16) and formula (17) represents height limit by flat and direct, flat and horizontal loaded mode; formula (18) represents the number of the $j$ th components; formula (19) represents the total number of components of transport; formula (20) represents the total number of PC components on the $i$ th vehicle; formula (21) represents that when the number of the transport vehicle loaded is larger than 0 , is 1 , namely, transportation vehicle is arranged.

\section{Application and Analysis}

Components list of a public rental housing project in one day are shown in table1.

Table1 Component List

\begin{tabular}{|c|c|c|c|c|c|c|c|c|c|c|c|}
\hline No. & Size(mm) & $\mathrm{Wt}(\mathrm{kg})$ & Qty & $\operatorname{Vol}\left(\mathrm{m}^{3}\right)$ & Lot & No. & Size $(\mathrm{mm})$ & $\mathrm{Wt}(\mathrm{kg})$ & Qty & $\operatorname{Vol}\left(\mathrm{m}^{3}\right)$ & Lot \\
\hline 1 & $2405 * 3430 * 60$ & 1185 & 1 & 0.495 & 2 & 19 & $4100 * 2865 * 320$ & 3507 & 4 & 3.759 & 1 \\
\hline 2 & $2405 * 3430 * 60$ & 1136 & 1 & 0.474 & 2 & 20 & $1200 * 2865 * 320$ & 1586 & 2 & 1.1 & 1 \\
\hline 3 & $2508 * 3430 * 60$ & 1238 & 1 & 0.516 & 2 & 21 & $2090 * 2865 * 320$ & 2762 & 2 & 1.916 & 1 \\
\hline 4 & $2047 * 4730 * 60$ & 1362 & 1 & 0.581 & 2 & 22 & $3090 * 2865 * 320$ & 4083 & 2 & 2.833 & 1 \\
\hline 5 & $2412 * 4730 * 60$ & 1624 & 1 & 0.685 & 2 & 23 & $4490 * 2865 * 320$ & 3891 & 1 & 4.116 & 1 \\
\hline 6 & $2412 * 4730 * 60$ & 1621 & 1 & 0.685 & 2 & 24 & $3160 * 2865 * 320$ & 4176 & 1 & 2.897 & 1 \\
\hline 7 & $2412 * 3330 * 60$ & 1140 & 1 & 0.482 & 2 & 25 & $5690 * 2865 * 320$ & 5139 & 1 & 5.217 & 1 \\
\hline 8 & $2412 * 4830 * 60$ & 1659 & 1 & 0.699 & 2 & 26 & $4300 * 2690 * 200$ & 4416 & 1 & 2.313 & 1 \\
\hline 9 & $2412 * 4830 * 60$ & 1656 & 1 & 0.699 & 2 & 27 & $4200 * 2690 * 200$ & 4315 & 5 & 2.26 & 1 \\
\hline 10 & $1200 * 2865 * 320$ & 1587 & 2 & 1.19 & 1 & 28 & $2720 * 1800 * 200$ & 1697 & 2 & 0.979 & 1 \\
\hline 11 & $3190 * 2865 * 320$ & 2629 & 3 & 2.925 & 1 & 29 & $2720 * 1500 * 200$ & 1410 & 1 & 0.816 & 1 \\
\hline 12 & $4870 * 2865 * 320$ & 3972 & 2 & 4.465 & 1 & 30 & $2720 * 3600 * 200$ & 3390 & 1 & 1.958 & 1 \\
\hline 13 & $2080 * 2830 * 320$ & 2635 & 2 & 1.884 & 1 & 31 & $2720 * 4000 * 200$ & 5291 & 1 & 2.176 & 1 \\
\hline 14 & $1700 * 2730 * 320$ & 1803 & 2 & 1.485 & 1 & 32 & $2720 * 3200 * 200$ & 2850 & 1 & 1.741 & 1 \\
\hline 15 & $2080 * 2830 * 320$ & 2205 & 2 & 1.884 & 1 & 33 & $2720 * 1900 * 200$ & 2514 & 1 & 1.034 & 1 \\
\hline 16 & $2370 * 2865 * 320$ & 3125 & 2 & 2.173 & 1 & 34 & $1810 * 1200 * 120$ & 266 & 1 & 0.261 & 3 \\
\hline 17 & $2090 * 2865 * 320$ & 2762 & 2 & 1.916 & 1 & 35 & $1810 * 1320 * 120$ & 293 & 1 & 0.287 & 3 \\
\hline 18 & $4100 * 2865 * 320$ & 3514 & 2 & 3.759 & 1 & 36 & $2776 * 1180 * 292$ & 2005 & 2 & 0.957 & 1 \\
\hline \multicolumn{6}{|c|}{$\mathrm{G}_{\mathrm{A}}=157220$} & \multicolumn{6}{|c|}{$\mathrm{N}_{\mathrm{A}}=58$} \\
\hline
\end{tabular}

With each household type in each apartment building with an independent gate being treated as a unit, the interior and exterior wallboards are firstly prefabricated; then comes to the composite boards and prefabricated air conditioning boards; finally key node pouring, bed mortar, and sleeve grouting are implemented. In order to meet the requirements of transportation, in the premise of meeting the construction process, the installation sequence can be adjusted properly. A standard layer of prefabricated total number is 58 , the total weight is $157220 \mathrm{~kg}$. According to the existing conditions, transport vehicles can choose 405 ShanQi Delong heavy truck, equipped with car size $11 \times 2.5 \mathrm{~m}$, rated load $40 \mathrm{t}, a=0.05 \mathrm{~m}, c=0.5 \mathrm{~m}$.

In the process of operation, the method of manual and computer interaction is used to calculate, according to the construction procedure, components in the same construction surface are divided into the same batch. According to the actual situation of this project, in order to simplify the calculation, in actual calculation process, to total volume of components to be installed in a certain period is to be $V_{A}$. Because the components of the fourth loading modes are less, we only consider the first three loading modes. There are also two rules needed to be considered: if $W_{i j k}>W, k=1$; if $j=j^{*}$, and $W_{i j k}<W, k=2$. Namely, if the width of the component is greater than the width of the transport vehicle, the component is transported by vertical and direct arrangement; if the component has a door and window hole, and component width is less than the width of the transportation vehicle, the loading way of the vertical and horizontal loading is adopted.

In this case, the component is divided into 3 batches, due to the two or three batches of the component is less, they can be transported in a transport vehicle, at the same time, the component of 
$12,23,25,35$ transferred to the transport vehicle. Without considering the transport of two or three batches, according to the existing transport scheme, the first batch of the required number of vehicles is not less than 5. According to the above optimization scheme for optimization, the required number of transport vehicle is optimized to be 4 .

According to the current transport scheme, transport these components need six car at least. By optimization, transport vehicle usage is reduced by $17 \%$, the utilization rate of vehicles is improved and the cost of transportation is reduced. Above instance is benchmarked against the standard layer of a public housing, the number of components in this case is fewer but the types is variety. It is conducive to improve the efficiency of truck loading. The optimized scheme is significant in reducing the transportation cost. If in large engineering application, the program will bring greater economic benefits to enterprise, and have more significant practical value in the transportation costs.

\section{Conclusions}

Starting from the goal of optimization of PC components of transportation cost, by mathematical programming, the loading scheme is optimized. Under the constraints of construction schedule, transport conditions, etc, the cost is minimized. The current situation of the low utilization rate and the single vehicle type selection of the PC components are changed. The influence of road condition of construction site or transport process on the vehicle model is considered, and the influence of different sizes of the components on the vehicle utilization is also considered. By comparative analysis, we can conclude that the optimized transportation scheme by above model is superior to the traditional scheme on transportation cost. The model is conducive to solving the problem of high cost of PC components transportation.

\section{Acknowledgements}

This work was supported by Humanities and Social Sciences Project from Ministry of Education of the People's Republic of China(15YJA630001); The Natural Science Foundation of Liaoning Province (201502794); Project from Ministry of Housing and Urban-Rural Development of the People's Republic of China(2014-R3-014); Science Research Program of Liaoning Province Education Administration: Liaoning city public safety management by E-CBR (W2014084) \& Research on the industry-university-research-user cooperative innovation mechanism of the market oriented research in Liaoning (W2014085); Work Safety Project of State Administration (2013-13) and Shenyang Scientific and Technological Planning (F15-198-5-15).

\section{References}

[1] Baoku Qi, Yang Zhang, Prefabricated construction development bottleneck and countermeasures research, Journal of Shenyang Jianzhu University (Social Science). 17(2015)156-159(In Chinese).

[2] Hengsong Liu, Yuanming Sun, Large-sized semicircular steel structures transportation technologies, Construction Technology. 42(2013) 211-213(In Chinese).

[3] Hongchun Wang, Construction logistics management based on bim technology, Technoeconomics \& Management Research . 12(2014) 55-58(In Chinese).

[4]Evensen, Knut. Advanced transportation management systems based on international standards. 21st World Congress on Intelligent Transport Systems: Reinventing Transportation in Our Connected World, ITSWC 2014, 2014.

[5] Lixin Zhou, Kun Liu. Intelligent logistics transportation system, Journal of Tongji University. 30(2002) 829-832(In Chinese).

[6] Yuan Fang, Zhaotang Wu. A study on the composition of construction logistics costs, Journal of Shenyang Jianzhu University (Social Science). 14(2012)376-391(In Chinese). 